\title{
Structure determination of tenofovir disoproxil (free base)
}

\author{
$\underline{\text { Preetam Anil }}^{1}$, Ruchira Sarbajna ${ }^{1}$, Suryanarayana Mulukutla ${ }^{1}$ \\ ${ }^{1}$ Analytical Development Laboratory, Mylan Laboratories Limited, Medak, India \\ E-mail: preetam.anil@mylan.in
}

Tenofovir disoproxil fumarate is a salt of an oral prodrug of Tenofovir. Tenofovir is a nucleoside monophosphate (nucleotide) analogue, a new class of agents, which has an in vitro antiviral activity against retroviruses and hepadnaviruses by inhibiting the reverse transcriptase enzyme hence, by DNA chain termination. Because Tenofovir was not well absorbed from the intestine, the prodrug, Tenofovir disoproxil, was developed to increase the bioavailability. The present study deals with the crystal structure of Tenofovir disoproxil which is a free base for Tenofovir disoproxil fumarate salt having the IUPAC name as $\operatorname{bis}(\{[($ propan-2-yloxy)carbonyl]oxy $\}$ methyl) (\{[(2R)-1-(6-amino-9H-purin-9-yl)propan-2-yl]oxy $\}$ methyl)phosphonate. To confirm the absolute configuration of the Tenofovir disoproxil (free base) crystallization experiments planned. Single crystals were developed from ethyl acetate solvent by slow evaporation method. The molecule crystallizes into orthorhombic with a non-centrosymmetric space group of P212121 having the cell parameters of $a=8.6960(3) \AA, b=12.2660(5) \AA, c=$ 23.8530(9) $\AA$. The molecule has strong inter and intra molecular hydrogen bonding which makes the molecule to remain intact. The intra molecular hydrogen bonding between $04 \ldots . . . .06$ leads to the formation cyclic five membered ring i.e., R11(5) and the inter molecular hydrogen bonding exists between N5...H6N...N4. The layers of stacked supra-molecular ions interact through hydrogen bonds ( N5...H6N...N4 ( $\left.(\mathrm{H} \ldots \mathrm{A})=2.22^{\circ} \mathrm{A}\right)$ stabilising the molecule inside the crystal lattice1,2. The molecular arrangement is in the form of helix which runs perpendicular to the $b$ - axis. The stereochemistry (absolute configuration) at one chiral center observed was confirmed as C (7)-R. It is published on Mylan Publication number: IPR/PR/2017/02.

[1] Giuseppe Resnati et al. (2015). IUCr], 2, 675-690

[2] Makinen, M., Nissinen, M., Rissanen, K. \& Vainiotalo, P. (2003). J.Am.Soc.Mass spec, 14, 143

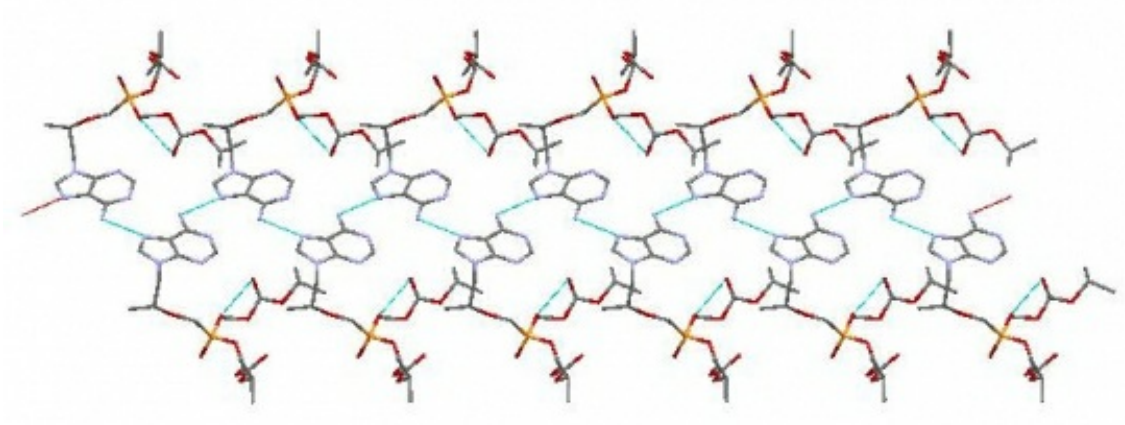

Keywords: Tenofovir disoproxil, Hydrogen bonding, supra molecular ions 